\title{
Solution Properties of Synthetic Polypeptides. IX. Light-Scattering Study of Poly-N5-(3-hydroxypropyl)-L-glutamine in the Helix-Coil Transition Region
}

\author{
Koichi OKita, ${ }^{*}$ Akio Teramoto, and Hiroshi Fujita \\ Department of Polymer Science, Osaka University, Toyonaka, Japan.
}

(Received June 29, 1970)

\begin{abstract}
Light-scattering measurements were carried out on fractions of poly$\mathrm{N}^{5}$-(3-hydroxypropyl)-L-glutamine (PHPG) in mixtures of methanol and water over ranges of temperature and solvent composition which covered the entire course of a helix-coil transition. Data for the mean-square radii of gyration $\left\langle\boldsymbol{S}^{2}\right\rangle$ were determined as a function of the parameters $N, u$, and $\sigma$ basic to the theories of the helix-coil transition of polypeptides, where $N$ is the number of monomeric units, $u$ the equilibrium constant for helix formation, and $\sigma$ the helix-initiation parameter. The data were analyzed in terms of the procedure proposed recently by Teramoto, et al., and it is shown that the molecular chain in the transition region could well be represented by an alternating sequence of helical sections characterized by a pitch of $1.65 \pm 0.05 \AA$ and randomly coiled sections represented by random flight chains with a step length of $15 \pm 0.5 \AA$. The value of $1.65 \AA$ is favorably compared to that for the $\alpha$-helix. These results lend support to the theory of Nagai for $\left\langle S^{2}\right\rangle$ and its subsequent extensions.

KEY WORDS Helix-Coil Transition / Mean-Square Radius of Gyration / Polypeptide / Light Scattering / Poly-N5-(3-Hydroxypropyl)L-glutamine /
\end{abstract}

An interesting and perhaps the most important problem in the investigation of molecular properties of polypeptides in dilute solution is concerned with the manner in which their average dimensions vary in the course of helix-coil transition. This problem was first treated theoretically by Nagai ${ }^{1}$ and subsequently by Miller and Flory ${ }^{2}$ and several others. ${ }^{3-5}$ Nagai obtained, subject to the neglect of excluded-volume effects, rigorous expressions for mean-square end-to-end distance $\left\langle R^{2}\right\rangle$ and mean-square radius of gyration $\left\langle S^{2}\right\rangle$ as functions of three basic parameters $N$, $u$, and $\sigma$ which govern the helix-coil transition and two size parameters $b_{0}$ and $b_{1}$ which characterize the contour lengths of randomly coiled and helical sections in a polypeptide molecule. Here $N$ is the number of peptide residues in the polypeptide chain, $u$ is the equilibrium constant for the formation of helix from random coil, and $\sigma$ is the helix-initiation (or cooperativity)

\footnotetext{
* Present address: Sumitomo Electric Industries Inc., Osaka, Japan.
}

parameter. Very recently, Teramoto, et al. ${ }^{6}$ have succeeded in simplifying the complicated expressions of Nagai for $\left\langle S^{2}\right\rangle$ and $\left\langle R^{2}\right\rangle$ into forms which are not only tractable for numerical calculations but are also readily utilizable for the analysis of experimental data for these quantities. Unfortunately, as far as we know, no actual data for $\left\langle S^{2}\right\rangle$ (note that $\left\langle R^{2}\right\rangle$ is not directly measurable) relevant to check the predictions from these theoretical studies are available in existing literature.

Recently, in connection with a study of PHPG (poly- $\mathrm{N}^{5}$-(3-hydroxypropyl)-L-glutamine), ${ }^{7}$ we have had an opportunity to perform extensive light-scattering measurements on this polypeptide in mixtures of water and methanol of various compositions and have already used part of the data for the arguments presented in Part $\mathrm{VI}^{7}$ of this series. In this paper, we wish to describe the details of these light-scattering measurements and, in conjunction with the data for $\sigma$ and helical contents reported in Part VI, examine how well the method of analysis proposed 
previously ${ }^{6}$ is applicable to the data for $\left\langle S^{2}\right\rangle$ obtained.

\section{EXPERIMENTAL SECTION}

\section{Polypeptide Samples}

Six fractions of PHPG, A-II, A-III, ..., A-VII, which had been prepared as described in Part VI, ${ }^{7}$ were selected for the present measurements. In order to find conditions in which reproducible light-scattering data could be obtained, the stability of PHPG solutions was examined in some detail. The results may be summarized as follows.

Right after the preparation all the fractions dissolved in pure methanol and in mixtures of methanol and water. However, after two weeks' storage at room temperature, some of them became partially insoluble in methanol. This trend was more conspicuous for higher molecular weight fractions and became more marked as time went on. Furthermore, the methanol solutions were not entirely stable at room temperature and the polypeptide gradually precipitated out of them upon standing. For samples A-II and A-III, heating did not bring the precipitated polymer back into solution but even enhanced the precipitation. When the insoluble polypeptide had been removed, the solution stayed stable for at least two days. Thus it appeared that the precipitation was a kind of crystallization process, which proceeded with a small amount of insoluble polypeptides as nuclei and was accelerated by heating. Applequist and Doty ${ }^{8}$ had reported a similar observation on poly-Llysine solutions.

Solutions of PHPG in mixtures of methanol and water were relatively stable and produced no insoluble fraction during the period when our light-scattering measurements were effected. Two years after the preparation, however, some of the fractions became insoluble even in water.

\section{Specific Refractive Index Increments}

A differential refractometer of the modified Schulz-Cantow type was used for determining the specific refractive index increments. The measurements were made at a variety of solvent conditions, covering the entire range of solvent composition and the temperature range between 5 and $40^{\circ} \mathrm{C}$.

\section{Light-Scattering Photometer}

Use was made of a Shimazu light-scattering photometer with cylindrical cells. The photometer was calibrated and the cell alignment was checked in the manner as described elsewhere. ${ }^{9}$ Data were taken at scattering angles between 35 and $135^{\circ}$ with unpolarized light of wavelength $4358 \AA$, and corrected for a back reflection of the incident beam by the method of Kratohvil. ${ }^{10}$ This correction had not been applied to the data reported in Part VI. ${ }^{7}$ Thus although the values of $\bar{M}_{w}$ reported therein are somewhat smaller than those given below, this hardly affects our previous arguments. ${ }^{7}$ Effect of the optical anisotropy of polypeptide solutions on the intensity of scattered light was examined with polarized light of wavelength $4358 \AA$ and found to be negligible.

\section{Procedure}

Methanol solutions were prepared by mixing known amounts of polypeptide and methanol in a stoppered flask. When an appreciable amount of insoluble polypeptide was observed, the solution was first passed through a sintered glass filter, centrifuged at $20000 \mathrm{~g}$ for one hour, and pipetted into the light-scattering cell. The solution in the cell was diluted directly with methanol which had been purified by centrifugation. The glass filter was washed quickly with methanol and dried in a vacuum oven to determine the amount of insoluble polypeptide residues. The concentration of the polypeptide was calculated from its original weight subtracted by that of the insoluble residues. The latter amounted to $10-13 \%$ of the former for samples A-I, A-II, and A-III, but was negligible for other samples. In some cases, concentrations were checked by dry-weight analysis of the solutions after light-scattering measurements had been completed and the results agreed with those by the above-mentioned method to an accuracy of $\pm 1 \%$.

For systems other than methanol solutions, centrifugation followed by filtration was necessary to purify the solutions before they were put in use for light-scattering measurements. The filtration apparatus used was fitted with Millipore filters of 0.3 micron pore size for aqueous $0.1-\mathrm{M}$ $\mathrm{NaCl}$ and with Gelman filters of the types $\alpha-6$, 
$\alpha-8$, and VF-6 for mixed solvents, and, before the polypeptide solution was passed through, it was thoroughly washed with a large volume of distilled water and then with about $100 \mathrm{ml}$ of the solvent mixture to be used. The cell was rinsed several times with a few milliliter of the filtered solution and then filled with about $30 \mathrm{ml}$ of it. Dilution was effected in the cell by adding the solvent mixture purified by centrifugation. Dry-weight analysis of the solutions showed no detectable change in polypeptide concentration before and after purification. Refractometry checked no change in solvent composition to occur during the purification process. Hence for systems other than methanol solutions we assumed the polypeptide concentrations and solvent compositions to be the same as in the original solutions.

The ratio $G_{35} / G_{90}$ was taken as a measure of purification, where $G_{35}$ and $G_{90}$ stand for the photometer readings at scattering angle 35 and $90^{\circ}$, respectively. We assigned that when the purification had been thoroughly made, this ratio would be approximately in the range from 2.5 to 2.8 for solvent mixtures and in the range from 2.9 to 4.0 for solutions, depending on the molecular weight of the polypeptide and solvent conditions. The filtration was repeated until this condition was fulfilled. With samples AII, A-III, and A-IV, use of a single filter sheet was sufficient, but for lower molecular weight samples it was necessary to use two or more sheets piled together.

\section{RESULTS}

Analysis of the Angular Distribution of the Intensity of Scattered Light

It is customary to express the reduced intensity $R_{\theta}$ of scattered light from a dilute polymer solution in the form

$$
\frac{K c}{\boldsymbol{R}_{\theta}}=\frac{1}{\bar{M}_{w} \boldsymbol{P}(\theta)}+\frac{2 A_{2} c P_{2}(\theta)}{\boldsymbol{P}(\theta)}+\cdots
$$

Here $K$ is the familiar optical constant, $\bar{M}_{w}$ the weight-average molecular weight of the dissolved polymer, $c$ its concentration, $\theta$ the scattering angle, $A_{2}$ the second virial coefficient of the system, and $P(\theta)$ and $P_{2}(\theta)$ the intra- and intermolecular scattering functions, respectively.
In the limit when $\theta$ approaches zero, both $\boldsymbol{P}(\theta)$ and $P_{2}(\theta)$ tend to unity, and eq 1 reduces to

$$
\frac{K c}{R_{0}}=\frac{1}{\bar{M}_{w}}+2 A_{2} c+\cdots
$$

At the limit of infinite dilution, eq 1 reduces to

$$
\left(\frac{K c}{R_{\theta}}\right)_{C=0}=\frac{1}{\bar{M}_{w} P(\theta)}=\frac{1}{\bar{M}_{w}}\left(1+\frac{1}{3} k^{2}\left\langle S^{2}\right\rangle+\cdots\right)
$$

with

$$
k=\frac{4 \pi}{\lambda^{\prime}} \sin \frac{\theta}{2}
$$

where $\left\langle S^{2}\right\rangle$ is the mean-square radius of gyration of the solute molecule and $\lambda^{\prime}$ the wavelength of light in the scattering medium.

Strictly speaking, the above equations are not applicable to interrupted helices in mixed solvents. A polypeptide molecule in the helix-coil transition region should be regarded as a copolymer consisting of helical and randomly coiled monomeric units, which may have different refractive index increments, $(\partial n / \partial c)_{1}$ and $(\partial n / \partial c)_{0}$. For such a polymer the direct application of eq 3 leads to a quantity which may be termed the apparent mean-square radius of gyration $\left\langle S^{2}\right\rangle_{\text {app }}$. Teramoto, et al., ${ }^{6}$ have shown that $\left\langle S^{2}\right\rangle_{\text {app }}$ is related to $\left\langle S^{2}\right\rangle$ by the equation

$$
\begin{aligned}
& \left\langle S^{2}\right\rangle_{\mathrm{app}}=\left\langle S^{2}\right\rangle\left(1+\delta f_{N}\right)^{-2} \\
& \quad \times\left\{1+\frac{\delta}{N^{2}\left\langle S^{2}\right\rangle_{i}} \sum_{i j}\left[2\left\langle r_{i j}^{2}\right\rangle_{10}+(2+\delta)\left\langle r_{i j}^{2}\right\rangle_{11}\right]\right\}
\end{aligned}
$$

with $\delta$ being defined by

$$
\delta=\frac{(\partial n / \partial c)_{1}}{(\partial n / \partial c)_{0}}-1
$$

As for the symbols appearing in eq 4 the reader should consult their original article. The extent to which $\left\langle S^{2}\right\rangle_{\text {app }}$ deviates from $\left\langle S^{2}\right\rangle$ is chiefly determined by the magnitude of $\delta$. A rough estimate of $\delta$ from the observed linear dependence of $\partial n / \partial c$ on helical content $f_{N}$ for PHPG fractions ${ }^{7}$ gives the following values: 0.38 for pure methanol at $15^{\circ} \mathrm{C}$ and 0.17 for water at $25^{\circ} \mathrm{C}$, both at the wavelength $4358 \AA$. Numerical calculations with $\delta=0.4$ show that the deviation is as small as $2 \%$, which is within the limits of experimental errors in $\left\langle S^{2}\right\rangle_{\text {app }}$. Therefore, in 


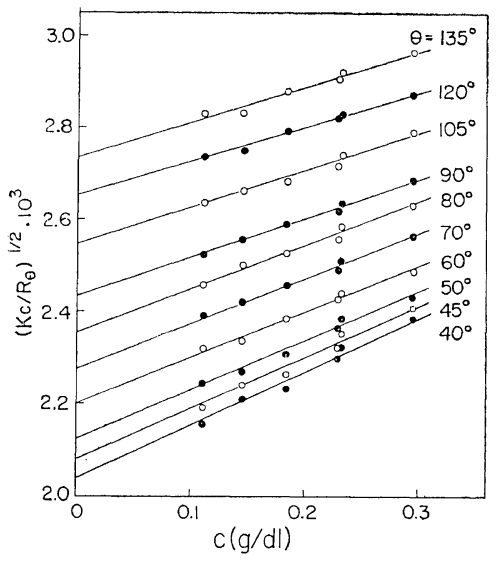

Figure 1. Concentration dependence of reciprocal scattering intensity for Sample A-III in methanol at $25^{\circ} \mathrm{C}$ at the indicated scattering angles.

what follows, we equate $\left\langle S^{2}\right\rangle_{\text {app }}$ to $\left\langle S^{2}\right\rangle$, discarding the possible effect of the copolymer nature of interrupted helices.

The analysis of light-scattering data is usually carried out by applying Zimm's procedure, ${ }^{11}$ in which $K c / R_{\theta}$ is plotted against $c$ or $\sin ^{2}(\theta / 2)$. Our experience with randomly coiled polymers ${ }^{9}$ has suggested, however, that a plot of $\left(K c / R_{\theta}\right)^{1 / 2}$, rather than $K c / R_{\theta}$, vs. $c$ facilitates a more reliable determination of $P(\theta)$. With the expectation that this would be the case with semi-flexible polymers such as polypeptides in the helix-coil transition region, we here use the square-root plot for all of our data. Figure 1 illustrates plots of this type with the data for sample AIII in methanol at $25^{\circ} \mathrm{C}$. It can be seen that each plot is fitted by a straight line and allows one to estimate the ordinate intercept with accuracy.

It has been demonstrated theoretically ${ }^{12}$ that a plot of $P(\theta)^{-1 / 2}$, rather than $P(\theta)^{-1}$, vs. $\sin ^{2}(\theta / 2)$ follows the initial tangent over a broader range regardless of molecular conformation, provided that the sample is reasonably homogeneous. On this ground we have plotted data for $P(\theta)$ in the form of $P(\theta)^{-1 / 2}$ vs. $\sin ^{2}(\theta / 2)$ and thereby estimated $\left\langle S^{2}\right\rangle$. Figure $2 \mathrm{a}$ shows a typical example of such a plot with the data for A-III taken from Figure 1. The plot has a linear portion long enough to determine its initial slope with fair precision. The solid line represents the theoretical curve for a monodisperse rod

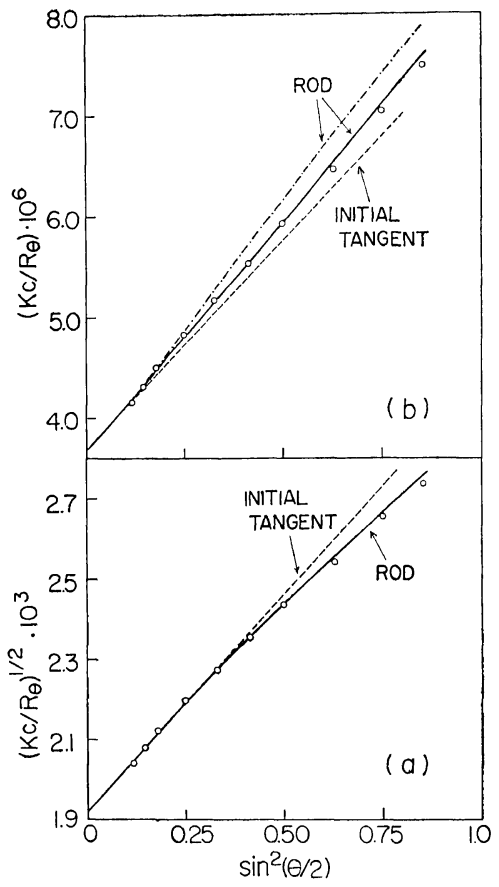

Figure 2. Representative data obtained by extrapolation to infinite dilution for Sample A-III in methanol at $25^{\circ} \mathrm{C}$ : (a) square-root plot and (b) Zimm plot. Solid lines represent the theoretical curves for a monodisperse rod and dotted lines are their initial tangents. The chain line represents the theoretical curve whose initial tangent fits the experimental data in the entire range on the $\mathrm{Zimm}$ plot.

consistent with this slope, and the experimental points are seen to follow it closely. Figure $2 b$ shows the same data on a plot of the Zimm type. As is evident from this figure, it is practically impossible to determine the correct initial tangent of a plot as shown here. The experimental points appear to follow a straight line over the entire range, and therefore one might be tempted to suppose this line to be a correct initial tangent and calculate $\left\langle S^{2}\right\rangle$ from its slope. The theoretical curve for a monodisperse rod corresponding to this slope, however, deviates largely upward from the experimental points. As shown in Table I, the values of $\left\langle S^{2}\right\rangle$ calculated from the plots of the Zimm type were about 10\% larger than those from the square-root plots. Although at present we are unable to decide which estimate is more correct, 
Table I. Comparison of square-root plot and Zimm-plot methods in terms of light-scattering data for samples $\mathrm{A}$-II and $\mathrm{A}-\mathrm{III}$ in methanol at $25^{\circ} \mathrm{C}$

\begin{tabular}{lccrcc}
\hline & \multicolumn{2}{c}{ Sample A-II } & & \multicolumn{2}{c}{ Sample A-III } \\
\cline { 2 - 3 } \cline { 5 - 6 } & Square-root plot & Zimm plot & & Square-root plot & Zimm plot \\
\hline $\bar{M}_{w} \times 10^{-4}$ & $36.6 \pm 0.5$ & $37.0 \pm 0.3$ & & $27.1 \pm 0.3$ & $27.0 \pm 0.2$ \\
$A_{2} \times 10^{-4}$, cgs & 2.40 & 2.65 & & 2.48 & 2.55 \\
$\left\langle S^{2}\right\rangle 1 / 2, \AA$ & $594 \pm 10$ & $629 \pm 10$ & & $476 \pm 10$ & $493 \pm 10$ \\
\hline
\end{tabular}

we believe that the values from the latter plots would be more reliable and have used them in the subsequent discussion.

\section{Data with Pure Methanol}

Figure 3 represents the resuts of PHPG fractions in methanol at $25^{\circ} \mathrm{C}$. The mean-square radii of gyration calculated from these data are summarized in Table II. Whenever possible, the limits of experimental error are indicated. Analysis of optical rotatory dispersion data ${ }^{7}$ has shown that PHPG is almost helical and assumes a rodlike shape in methanol at $25^{\circ} \mathrm{C}$. In fact, it is possible to fit the theory of monodisperse rods to the data of Figure 3. The lengths $h$ per monomeric residue as calculated from $\left\langle S^{2}\right\rangle$ by assuming a straight rod for the molecular shape scatter around $1.1 \AA$, which is much smaller than that expected for the $\alpha$-helix. An $h$ of this magnitude may be obtained, provided that PHPG in methanol contains one or two breaks in the molecule. In this connection, it is instructive to note that our previous data indicated that even in this helix-forming solvent PHPG still contains a considerable number of randomly coiled residues. ${ }^{7}$ Miller and Flory $^{2}$ made a similar suggestion to account for a gradual decrease in $h$ of poly( $\gamma$-benzyl-L-glutamate) (PBLG) in helicogenic solvents with increasing molecular weight. For poly- $\varepsilon$-carbobenzoxy-Llysine (PCBL) in its helicogenic solvent dimethylformamide Applequist and Doty ${ }^{8}$ obtained $h$ values much smaller than $1.5 \AA$ and attempted

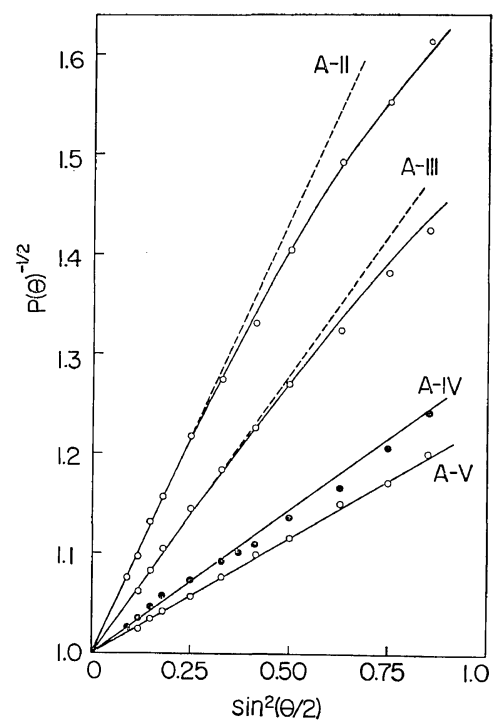

Figure 3. Typical plots of $P(\theta)^{-1 / 2} v s . \sin ^{2}(\theta / 2)$ for fractions of PHPG in methanol at $25^{\circ} \mathrm{C}$. Solid lines represent the theoretical curves for monodisperse rods and dotted lines are their initial tangents.

Table II. Light-scattering data for fractions of PHPG in pure methanol at $25^{\circ} \mathrm{C}$

\begin{tabular}{ccccc}
\hline Sample code & $\bar{M}_{w} \times 10^{-4}$ & $A_{2} \times 10^{4}, \mathrm{cgs}$ & $\left\langle S^{2}\right\rangle^{1 / 2}, \AA$ & $h$, $^{\mathrm{a}} \AA$ \\
\hline A-II & $36.6 \pm 0.5$ & 2.40 & $594 \pm 10$ & $1.05 \pm 0.03$ \\
A-III & $27.1 \pm 0.3$ & 2.48 & $476 \pm 10$ & $1.13 \pm 0.03$ \\
A-IV & 22.2 & 3.0 & $343 \pm 7$ & $1.00 \pm 0.03$ \\
A-V & 16.8 & $3.0 \pm 0.1$ & $320 \pm 10$ & $1.23 \pm 0.05$ \\
A-VI & $10.0 \pm 0.5$ & $4.1 \pm 0.6$ & $210 \pm 15$ & $1.35 \pm 0.1$ \\
A-VII & $5.5 \pm 0.1$ & 3.3 & $(83 \pm 5)^{\mathrm{b}}$ & $(0.97 \pm 0.12)^{\mathrm{b}}$ \\
\hline
\end{tabular}

${ }^{a} h$, pitch per monomeric residue calculated by assuming a straight rod for the molecular conformation. ${ }^{\mathrm{b}}$ The values in the parenthese are less reliable. 


\section{Light-scattering from Polypeptide Solutions}

to interpret them in terms of the wormlike chain model. However, they were led to a contradictory conclusion that the contour length of the polypeptide chain would not be proportional to molecular weight. In order to cast light on this contradiction, we now plan to perform a light-scattering study on PCBL.

\section{Data with Mixed Solvents}

Figure 4 shows plots of $\left(K c / R_{\theta}\right)^{1 / 2}$ vs. $c$ for sample A-II in mixtures of methanol and water at $25^{\circ} \mathrm{C}$. The plots at different solvent compositions do not converge to a common ordinate intercept. The divergence appears to be beyond the limits of experimental error. It is unlikely that the molecular weight of a polypeptide may depend on solvent under the conditions of the present experiments. We may rule out the possibility that the preferential absorption of either water or methanol on the polypeptide molecule could account for the divergence, because these liquids are relatively good solvents for PHPG and have similar refractive indices. For the moment we regard the values of $\bar{M}_{w}$ determined in methanol solutions as correct ones, since they are consistent with those determined previously from separate sedimentation equilibrium experiments. ${ }^{7}$

Figure 5 shows plots of $P(\theta)^{-1 / 2} v s \cdot \sin ^{2}(\theta / 2)$ for sample $\mathrm{A}-\mathrm{II}$ in various mixtures of methanol and water at $25^{\circ} \mathrm{C}$. The appreciable increase in the initial slopes of the plots with increasing methanol content is indicative of the transition from helix to coil. Features of these plots are

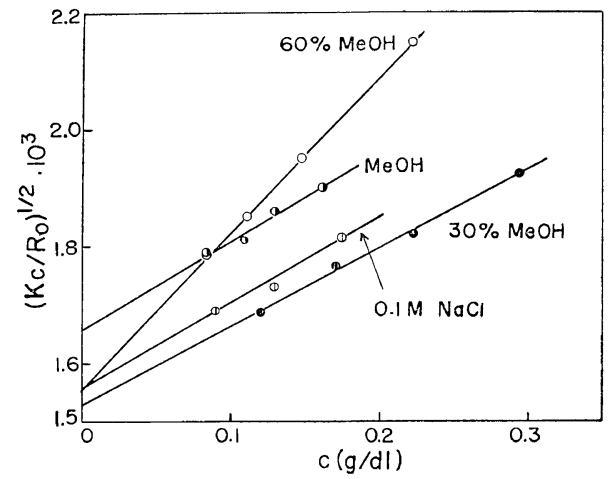

Figure 4. Representative data obtained by extrapolation to zero angle for Sample A-II in mixtures of methanol and water at $25^{\circ} \mathrm{C}$. substantially similar to those which have been calculated according to an approximate equation ${ }^{12}$ (cf. Figure 4 in ref 12). It appears that the data for 60 -wt $\%$ methanol as well as those for

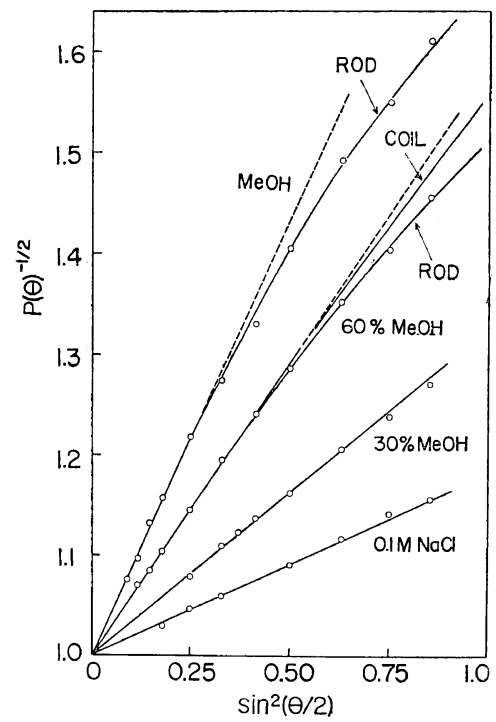

Figure 5. Representative data obtained by extrapolation to infinite dilution for Sample A-II in mixtures of methanol and water at $25^{\circ} \mathrm{C}$. Solid lines represent the theoretical values for monodisperse coils or rods, and dotted lines are their initial tangents.

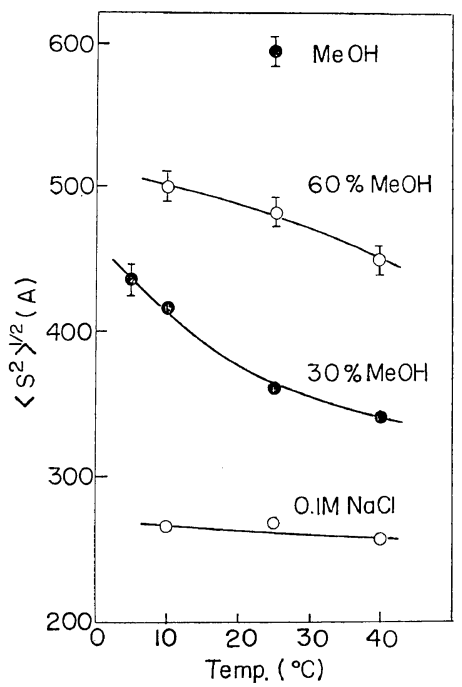

Figure 6. Dependence of the root-mean-square radius of gyration on temperature for Sample A-II in the indicated solvents. 


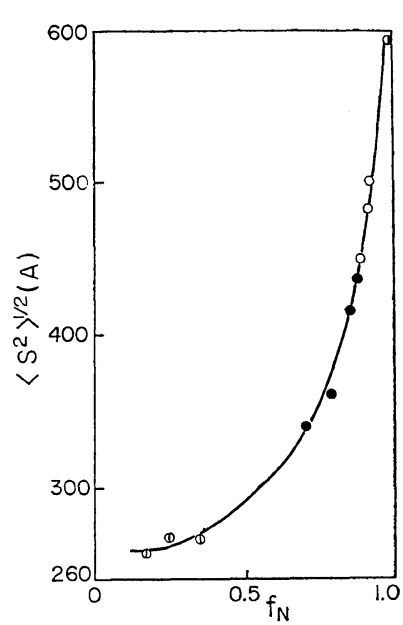

Figure 7. Dependence of root-mean-square radius of gyration on helical content for PHPG A-II in mixtures of methanol and water: pure methanol, 1) 60-wt $\%$ methanol, $\bigcirc$; 30-wt $\%$ methanol, $0.1 \mathrm{M}$ aqueous $\mathrm{NaCl}$, (1).

pure methanol are fitted reasonable well by theoretical curves for the monodisperse rods which are consistent with the corresponding initial slopes. However, the conformation of PHPG in the former solvent has been shown to be far from being perfectly helical. ${ }^{7}$ In view of this confusing result, it is hazardous to draw a conclusion about the molecular conformation from comparison of an experimentally determined shape of $P(\theta)$ with theory.

Figure 6 illustrates, with the data for A-II, how $\left\langle S^{2}\right\rangle^{1 / 2}$ changes with temperature and solvent composition. Vertical segments attached to the plotted points represent the uncertainties involved in the graphical determination of $\left\langle S^{2}\right\rangle^{1 / 2}$, which are within several percent in most cases. The root-mean-square radius of gyration of $\mathrm{A}-\mathrm{II}$ is seen to decrease with raising temperature most sharply at the methanol content of $30-w t \%$, where the helical content nearly approaches the helix side. ${ }^{7}$ In Figure 7 these data are replotted against the values of helical content $f_{N}$ taken from the previous paper. ${ }^{7}$ The features of this

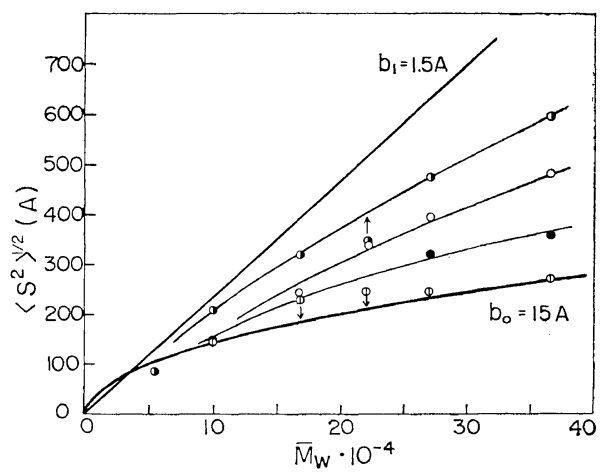

Figure 8. Dependence of the root-mean-square radius of gyration on molecular weight for fractions of PHPG at $25^{\circ} \mathrm{C}$ in pure methanol (()), 60-wt $\%$ methanol ( $\bigcirc$ ), 30-wt $\%$ methanol $(\bullet)$, and $0.1 M$ aqueous $\mathrm{NaCl}$ (1)). The upper thick line indicates the theoretical relation for straight rods corresponding to the $\alpha$-helix, and the lower one that for random flight chains with a step length of $15 \mathrm{~A}$.

Table III. Data for $\left\langle S^{2}\right\rangle^{1 / 2}$ of PHPG fractions in mixtures of methanol and water ${ }^{2}$

\begin{tabular}{|c|c|c|c|c|c|c|}
\hline Solvent & Temp, ${ }^{\circ} \mathrm{C}$ & $\mathrm{A}-\mathrm{II}$ & A-III & $A-I V$ & $A-V$ & $A-V I$ \\
\hline $60-w t \%$ & 10 & $500 \pm 10$ & $409 \pm 5$ & - & - & - \\
\hline \multirow[t]{2}{*}{ Methanol } & 25 & $482 \pm 10$ & $396 \pm 5$ & 339 & $245 \pm 5$ & $148 \pm 10$ \\
\hline & 40 & $449 \pm 10$ & $376 \pm 10$ & - & - & - \\
\hline $30-w t \%$ & 5 & $436 \pm 10$ & $373 \pm 7$ & - & - & - \\
\hline \multirow[t]{3}{*}{ Methanol } & 10 & $415 \pm 5$ & $340 \pm 5$ & - & - & - \\
\hline & 25 & $360 \pm 5$ & $323 \pm 2$ & - & $240 \pm 5$ & $144 \pm 5$ \\
\hline & 40 & $340 \pm 4$ & $293 \pm 4$ & - & - & - \\
\hline \multirow[t]{4}{*}{$0.1-M \mathrm{NaCl}$} & 5 & - & $258 \pm 10$ & - & - & - \\
\hline & 10 & $266 \pm 5$ & $239 \pm 5$ & - & $233 \pm 5$ & - \\
\hline & 25 & $268 \pm 5$ & $246 \pm 10$ & $249 \pm 5$ & $230 \pm 5$ & $145 \pm 5$ \\
\hline & 40 & $257 \pm 5$ & $224 \pm 5$ & - & $219 \pm 5$ & - \\
\hline
\end{tabular}

${ }^{a}\left\langle S^{2}\right\rangle^{1 / 2}$ is expressed in angstrom units. The uncertainties involved in the graphical determination of $\left\langle S^{2}\right\rangle^{1 / 2}$ are indicated. 
curve are quite similar to those of the theoretical curves calculated by Nagai $^{1}$ and by Miller and Flory. $^{2}$ The curve also bears resemblance to those of $[\eta] v s . f_{N}$ (cf. Figure 9 in ref 7), indicating a close correlation to exist between $[\eta]$ and $\left\langle S^{2}\right\rangle$.

Figure 8 demonstrates the molecular weight dependence of $\left\langle S^{2}\right\rangle^{1 / 2}$ at $25^{\circ} \mathrm{C}$ and fixed solvent compositions. For reference two thick lines are drawn; the upper one represents the theoretical values for straight rods consisting of the $\alpha$-helix, and the lower corresponds to those for random flight chain with a step length of $15 \AA$. The latter line appears to fit the data for aqueous $0.1 M-\mathrm{NaCl}$, in which the molecular conformation has been shown from ORD data ${ }^{7}$ to be partially helical rather than randomly coiled. The values for $\left\langle S^{2}\right\rangle^{1 / 2}$ in methanol is considerably lower than those expected for the $\alpha$-helix. Table III summarizes the numerical values of $\left\langle S^{2}\right\rangle^{1 / 2}$ obtained in mixed solvents.

\section{DISCUSSION}

At intermediate stages of a helix - coil transition, the polypeptide chain may consist of alternating sequences of helical and randomly coiled sections and thus assumes a conformation generally termed an interrupted helix. In his calculation of the molecular dimensions of interrupted helices, $\mathrm{Nagai}^{1}$ replaced a helical section consisting of $n_{1}$ monomeric residues by a straight rod of $n_{1} b_{1}$ in length and a randomly coiled section consisting of $n_{0}$ monomeric residues by a random flight chain of $n_{0}$ steps, each of which is $b_{0}$ long, and connected the two sections by flexible joints. Very recently, Teramoto, et al., ${ }^{6}$ have shown that Nagai's exact equation for $\left\langle S^{2}\right\rangle$ of such a model chain can be simplified to a form tractable for the analysis of experimental data. It reads

$$
\frac{H_{1}}{H_{2}}=b_{0}{ }^{2}+\frac{H_{3}}{H_{2}} b_{1}{ }^{2}
$$

where

$$
\begin{gathered}
H_{1}=\frac{\left\langle S^{2}\right\rangle}{(1 / 6) N(1-f)} \\
H_{2}=1+\frac{12 f^{2}}{\beta^{2}}\left[1-\frac{2 \sqrt{f(1-f)}}{\beta}\right]
\end{gathered}
$$

$$
H_{3}=\frac{2}{\sqrt{\sigma}}\left(\frac{f}{1-f}\right)^{3 / 2} Q(\beta, f)
$$

and

$$
\begin{aligned}
Q(\beta, f)= & 1-\frac{3}{\beta}\left(\frac{f}{1-f}\right)^{1 / 2} \\
& +\frac{6}{\beta^{2}}\left(\frac{f}{1-f}\right)\left[1-2(1-f)^{2}\right] \\
& -\frac{6}{\beta^{3}}\left(\frac{f}{1-f}\right)^{3 / 2}\left[1-2(1-f)^{2}(3-2 f)\right. \\
& -\frac{(2 f-1)^{2}}{f^{4}} \exp \left(-\beta \sqrt{\left.\frac{1-f}{f}\right)}\right] \quad(10)
\end{aligned}
$$

Here $f$ stands for the helical content for infinite $N$ and $\beta=N \sqrt{\sigma}$.

Granted that $b_{0}$ and $b_{1}$ stay constant throughout the transition, then it follows from eq 6 that a plot for $H_{1} / H_{2}$ vs. $H_{3} / H_{2}$ should give a straight line, whose ordinate intercept and slope are $b_{0}{ }^{2}$ and $b_{1}{ }^{2}$, respectively. The values of $f$ and $\sigma$ needed to compute $H_{2}$ and $H_{3}$ are obtainable from the analysis of ORD data in terms of the procedure described elsewhere. ${ }^{7}$ Thus the combination of light-scattering data with ORD data will enable a test of the validity of eq 6 .

Values of $H_{1}, H_{2}$, and $H_{3}$ were calculated for samples A-II and A-III, with the data taken from Table III of this paper and Table II of

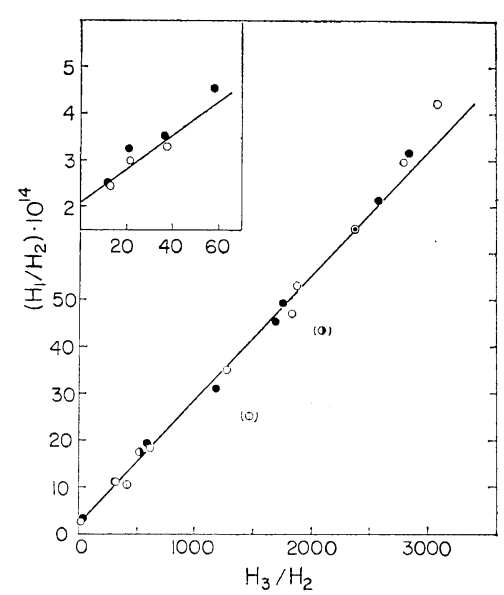

Figure 9. Analysis of the $\left\langle S^{2}\right\rangle$ data for fractions of PHPG in various mixtures of methanol and water in terms of the proposed procedure (see text): A-II, $\bigcirc$; A-III, ๑; A-IV, $\odot$; A-V, (1); A-VI, C. Insert gives an enlarged plot at smaller values of $H_{3} / H_{2}$. 
Part VI. ${ }^{7}$ Figure 9 shows the resulting plots of $H_{1} / H_{2}$ vs. $H_{3} / H_{2}$. The data for pure methanol have been omitted, because the values for $f$ in this system exceeded the limits of the validity of eq 6. The insert gives an enlarged plot at smaller values of $\mathrm{H}_{3} / \mathrm{H}_{2}$, which serves to determine the ordinate intercept with precision. It can be seen that the data points closely follow a straight line and substantiate the validity of eq 6 (subject to constant $b_{0}$ and $b_{1}$ ). The straight line yields $b_{0}=15.0 \pm 0.5 \AA$ and $b_{1}=1.65 \pm$ $0.05 \AA$. The latter compares favorably with $1.5 \AA$ characteristic of the $\alpha$-helix, but the small difference may deserve further comments. No experimental evidence is as yet reported for the helical structures of polypeptides in solution other than the $\alpha$-helix. We are therefore tempted to attribute the difference either to the polydispersity of the polypeptide samples studied or to the excluded-volume effect. Both of these possibilities may be equally probable, since, on one hand, the $P(\theta)$ curves for samples A-II and A-III shown in Figures 3 and 5 appear to have features characteristic of polydisperse samples and, on the other hand, the second virial coefficients of the systems examined were as large as $(2-3) \times 10^{-4}(\mathrm{cgs})$ in the entire range of the transition. The value of $15 \AA$ for $b_{0}$ is larger than $11.4 \AA$ deduced for unperturbed polypeptide chains by Brant and Flory, ${ }^{13}$ but smaller than $26 \AA$ calculated from the data ${ }^{14}$ for PBLG in DCA. The excluded-volume effects may be a dominant factor for the disparity among these $b_{0}$ values. Apart from such problems, we may conclude that the results presented above lend support to Nagai's theory ${ }^{1}$ and subsequent developments ${ }^{2-5}$ on the average dimensions of polypeptide chains in the helix-coil transition region.

\section{Remarks}

In qualitative terms, the predictions of these theories on the mean-square radius of gyration of a polypeptide molecule have been well borne out by our recent viscometric studies on poly$(\gamma \text {-benzyl-L-glutamate })^{15}$ and on poly( $\beta$-benzyl-Laspartate $)^{16}$ as far as the dependence on helical content and on the parameter $\sigma$ is concerned. However, a quantitative discussion of such viscosity data must be reserved until we can establish a relationship between $\left\langle S^{2}\right\rangle$ and [ $\eta$. This type of work is inviting in connection with the exploration of the molecular mechanisms of hydrodynamic properties of semiflexible polymers in dilute solution.

Acknowledgment. Thanks are due to $\mathrm{Mr}$. T. Norisuye for his discussion.

\section{REFERENCES}

1. K. Nagai, J. Chem. Phys., 34, 887 (1961).

2. W. G. Miller and P. J. Flory, J. Mol. Biol., 15, 298 (1966).

3. M. Go, N. Saito, and M. Ochiai,, J. Phys. Soc. Japan, 22, 227 (1967).

4. T. M. Birshtein and O. B. Ptitsyn, "Conformations of Macromolecules," John Wiley \& Sons, Inc. New York, N.Y., 1966, Chapter 9.

5. O. B. Ptitsyn, in "Conformation of Biopolymers." G. N. Ramachandran Ed., Academic Press Inc., New York, N.Y., 1967, p 381.

6. A. Teramoto, T. Norisuye, and H. Fujita, Polymer J., 1, 55 (1970).

7. K. Okita, A. Teramoto, and H. Fujita, Biopolymers, 9, 717 (1970).

8. J. Applequist and P. Doty, in "Polyamino Acids, Polypeptides, and Proteins," M. A. Stahman Ed., The University of Wisconsin Press, Wisconsin, 1961, p 161.

9. T. Norisuye, K. Kawahara, A. Teramato, and H. Fujita, J. Chem. Phys., 49, 4330 (1968).

10. J. P. Kratohvil, J. Colloid Interface Sci., 21, 498 (1966).

11. B. H. Zimm, J. Chem. Phys., 16, 1099 (1948).

12. T. Norisuye and A. Teramoto, Polymer J., 1, 341 (1970).

13. D. A. Brant and P. J. Flory, J. Amer. Chem. Soc., 87, 2788 (1965).

14. P. Doty, J. H. Bradbury, and A. M. Holtzer, J. Amer. Chem. Soc., 78, 947 (1956).

15. A. Teramoto, K. Nakagawa, and H. Fujita, J. Chem. Phys., 46, 4197 (1967).

16. Y. Hayashi, A. Teramoto, K. Kawahara, and H. Fujita, Biopolymers, 8, 403 (1969). 